\title{
Creation of Educational Resources: A Research Scientist's Role
}

\author{
Carol A. Christian \\ Space Telescope Science Institute, 3700 San Martin Drive, Baltimore, \\ $M D 21218$ USA
}

\begin{abstract}
Material and resources for use in science education must contain accurate, up-to-date data and research results. Clearly when curricula and other materials for use in informal science education and for public understanding of science are developed, the direct interaction and influence of research scientists is beneficial. What is the role of scientists in resource development? In colleges and universities, educational materials are scientist-centric since scientists are the principal science educators and therefore create the specific courseware they need. In a pre-college educational environment, and in science museums (for example), appropriate product creation is driven by experienced educators and other experts. The research scientist, drawn from a research environment, may not be the best instigator of those resources.
\end{abstract}

\section{Introduction}

At the Space Telescope Science Institute (STScI), we are responsible for providing the science operations for the Hubble Space Telescope (HST), and in the future, the Next Generation Space Telescope. Scientists are integrated into every functional area of the observatory, contributing scientific insight to guide the operations, data analysis, archiving and the software development for Hubble and our other missions. Our goal was to sensibly incorporate scientists into the construction of informational (news), educational and outreach resources that we offer to the public. We appreciate that the expectation for the quality and reliability of resources produced by Hubble and other NASA missions is quite high and therefore we devoted considerable attention to the techniques used to produce materials for our wide audiences.

When we initiated the education and outreach program several years ago, the role of the research scientist was not well defined, and was a relatively new function in the observatory setting. This paper describes the model we evolved after several other methods were tested.

\section{The Model}

In developing the model described here, we observed and directly experienced a number of scenarios for resource development with varying success. We noted that in circumstances where the scientist was involved as a consultant, often 
located at another institution, resource creation progressed stochastically, with many revisions and corrections being required. As a result, schedules slipped and the scientific integrity of the content was compromised. In a symmetric situation, when some of the materials production was contracted out (such as in online resource creation), similar difficulties were encountered.

We found that bringing scientists into a team environment, though challenging, was an optimal scenario. Teams are comprised of writers, programmers, web developers, graphic artists, educators, evaluators, and any other individuals with expertise relevant to the particular material being created. Teams are lead by an experienced production chief or any other individual talented in maintaining ambitious schedules as well as a familiarity with the target audiences (pre-college teaching, science museums, news media). We found that scientists could drive production forcefully, but the resource developed might not meet the "end user's" needs as well as we desired.

\subsection{Public Science Awareness and News}

We realize that for our scientific endeavors such as astronomy research to win public support, we must assist scientists in reporting their investigative results to the public through the news media. Researchers bring science results based on their HST data to the STScI news team and the news chief responsible for the crafting of materials appropriate for the news media and journalists based on those results. The rest of the news team is comprised of at least a science writer, a graphic artist and a visualization expert. An "outreach scientist" (a staff research scientist skilled in science communications, education and outreach) serves as a peer advocate for the investigator bringing forward the new results and works with the news team on a daily basis. The outreach scientist and the researcher collaborate with the team on background materials and ancillary information required for a release. Scientists provide the core information for caption and press release text that is written by the science writer. They collaborate on the visualization of the data and the astrophysical phenomena being showcased.

\subsection{Pre-college Curriculum Support Materials}

The creation of curriculum support materials requires keen attention to the national education standards. In addition, if state, regional or local standards or requirements need to be met, then curriculum experts and knowledgable educators are critical to the team. Teachers with classroom experience are vital for construction of usable materials. The creation and production process is quite extensive as described by Christian et al. (2001).

Scientists collaborate with the team to provide scientific data, insight on the latest relevant investigations, and to continually check on scientific accuracy. In our model, the team consists of artists, programmers, writers, educators with curriculum and standards expertise, teachers, an evaluator and web developers. The creation of the resources involves a design and production schedule as follows: goal description, concept design, story boarding of the resource, draft construction, review, production, testing, review, revision, initial release, review and evaluation. Scientists are responsible at all stages for scientific content, accuracy, currency and integrity. Teams are lead initially by an individual with 
development expertise and often at the production stage, the resource is handed off to an experienced production chief.

As described by Christian et al. (2001), the process may seem elaborate, but the reward is that robust, high quality professional resources are made available to teachers and students.

\subsection{Informal Science Education}

Similar to the development of pre-college educational materials, scientists participate in design teams for museum exhibits. They are contributors in the audience definition stage. They naturally play a principal role in the content specification for the exhibit. Designers lead the crafting of the exhibit presentation including physical materials, models, interactive units, multi-media, tactile displays, textual presentation and graphical elements. Researchers are responsible for providing the underlying science of the exhibit and also must be vigilant to explain concepts to team members repeatedly to insure accuracy. We found that including one of our own outreach scientists on the team worked effectively. That individual could in turn broker information supplied by research collegues who would be available only sporadically.

Scientists experienced in communicating with the public assist in identifying pre-conceptions that the target audience might have before experiencing the exhibit. They are involved in the prototype in situ testing. Scientists provide descriptions of technical details such as scientific instrument construction, telescope fabrication and data reduction techniques. For example, in the Hubble Space Telescope Traveling Exhibit New Views of the Universe, scientists provided the underlying modeling code to create an interactive on galaxy collisions (c.f., http://hubble.stsci.edu/discoveries/hstexhibit/index.html) and the description of how scientific ideas are developed into observing programs and subsequent research and publication.

\subsection{Public Information}

Website development is accomplished through a team effort, usually led by an experienced web developer or production manager. Scientists collaborate on all aspects: audience definition, content definition, storyboarding, prototypes, testing, review, revision and evaluation. Other key team members are graphic artists, writers and multimedia experts. An educator, usually one skilled in informal science education is often on the team as well.

\section{Summary}

We developed a team-based model for involving research scientists in the creation of the plethora of educational and outreach materials produced in support of the Hubble Space Telescope Mission. We found that we could achieve success through teams lead by experienced production chiefs working closely with a team including a local outreach scientist. Other scientists contribute sporadically to the content, and their participation is brokered by the outreach scientist. The model worked in preference to other scenarios enabling us to produce the high quality materials the public has grown to expect. 
Acknowledgments. This work is supported by a contract, NAS5-26555, to the Association of Universities for Research in Astronomy, Inc. for the operation of the Hubble Space Telescope at Space Telescope Science Institute. The input and experience of numerous individuals especially in the Office of Public Outreach, is acknowledged.

\section{References}

Christian, C.A., Eisenhamer, B., Eisenhamer, J., \& Teays, T. 2001, Journal of Science and Technology Education, 10, 38 